Edith Cowan University

Research Online

Research outputs 2014 to 2021

$12-1-2021$

\title{
Nano-grating assisted light absorption enhancement for msm-pds performance improvement: An updated review
}

Narottam Das

Mohammad Nur-E-alam

Edith Cowan University

Alif Islam

Ain Zulaikha Maslihan Ain

Follow this and additional works at: https://ro.ecu.edu.au/ecuworkspost2013

Part of the Physical Sciences and Mathematics Commons

10.3390/photonics8120539

Das, N., Nur-E-Alam, M., Islam, A., \& Ain, A. Z. M. (2021). Nano-grating assisted light absorption enhancement for msm-pds performance improvement: An updated review. Photonics, 8(12), article 539.

https://doi.org/10.3390/photonics8120539

This Journal Article is posted at Research Online.

https://ro.ecu.edu.au/ecuworkspost2013/11724 


\title{
Nano-grating Assisted Light Absorption Enhancement for MSM-PDs Performance Improvement: An Updated Review
}

\author{
Narottam Das ${ }^{1,2, *(\mathbb{D}}$, Mohammad Nur-E-Alam ${ }^{1,3}{ }^{\oplus}$, Alif Islam ${ }^{4}\left(\mathbb{C}\right.$ and Ain Zulaikha Maslihan Ain ${ }^{5}$ \\ 1 School of Engineering and Technology, Central Queensland University, Melbourne Campus, \\ Melbourne, VIC 3000, Australia; m.nur-e-alam@ecu.edu.au \\ 2 Centre for Intelligent Systems, School of Engineering and Technology, Central Queensland University, \\ Brisbane Campus, Brisbane, QLD 4000, Australia \\ 3 School of Science, Edith Cowan University, Joondalup, WA 6027, Australia \\ 4 Department of Electrical and Electronic Engineering, Southeast University, Dhaka 1213, Bangladesh; \\ alif.islam@seu.edu.bd \\ 5 NXP Semiconductors, Product Test Division, Petaling Jaya 47300, Selangor, Malaysia; juemaslihan@gmail.com \\ * Correspondence: n.das@cqu.edu.au
}

Citation: Das, N.; Nur-E-Alam, M.; Islam, A.; Ain, A.Z.M. Nano-grating Assisted Light Absorption Enhancement for MSM-PDs Performance Improvement: An Updated Review. Photonics 2021, 8, 539. https://doi.org/10.3390/ photonics 8120539

Received: 12 November 2021 Accepted: 26 November 2021 Published: 28 November 2021

Publisher's Note: MDPI stays neutral with regard to jurisdictional claims in published maps and institutional affiliations.

Copyright: (c) 2021 by the authors. Licensee MDPI, Basel, Switzerland. This article is an open access article distributed under the terms and conditions of the Creative Commons Attribution (CC BY) license (https:// creativecommons.org/licenses/by/ $4.0 /)$.

\begin{abstract}
The primary focus of this review article mainly emphasizes the light absorption enhancement for various nanostructured gratings assisted metal-semiconductor-metal photodetectors (MSMPDs) that are so far proposed and developed for the improvement of light capturing performance. The MSM-PDs are considered as one of the key elements in the optical and high-speed communication systems for applications such as faster optical fiber communication systems, sensor networks, high-speed chip-to-chip interconnects, and high-speed sampling. The light absorption enhancement makes the MSM-PDs an ideal candidate due to their excellent performances in detection, especially in satisfying the high-speed or high-performance device requirements. The nano-grating assisted MSM-PDs are preordained to be decorous for many emerging and existing communication device applications. There have been a significant number of research works conducted on the implementation of nano-gratings, and still, more researches are ongoing to raise the performance of MSM-PDs particularly, in terms of enhancing the light absorption potentialities. This review article aims to provide the latest update on the exertion of nano-grating structures suitable for further developments in the light absorption enhancement of the MSM-PDs.
\end{abstract}

Keywords: nano-grating; metal-semiconductor-metal photodetector; light absorption enhancement; absorption coefficient; opto-electronic devices

\section{Introduction}

Photodetectors are devices that transform optical impulses into electrical signals and are critical in many current optoelectronic applications. Photoconductors, photodiodes, avalanche photodiodes, Schottky junctions, and metal-semiconductor-metal photodetectors (MSM-PDs) are among the most prevalent types of photodetectors used in current technological applications [1-12]. An MSM-PD consists of two interdigitated metallic electrodes on the top surface of a semiconductor layer (e.g., Schottky contacts). When an external voltage is applied to the electrodes, one of the Schottky diodes is biased forward while the other one is biased backward. In between the electrodes on the semiconductor, incident light creates carriers that are drifted by the electric field (which is a pure drift photocurrent) as there is no diffusion component to slow down the device response [6-17]. The notion of an MSM-PD was first presented in 1979, and various research groups later reported the actual production of MSM-PDs [6,18-21]. The MSM-PD has unique characteristics, such as high optical light sensitivity and facile and cost-effective manufacture on a variety of substrates such as $\mathrm{Si}, \mathrm{SiO}_{2}$, and $\mathrm{GaAs}$, making them an excellent choice for application in current ultra-high-speed communication systems [4-18,21,22]. 
The MSM-PDs have a number of benefits in practical application. They have an extremely quick photoresponse, which is dictated by the carriers' saturation velocity. There are no Ohmic connections required, allowing for the low-doped active material. Due to the planar geometry's low capacitance, the RC time constant is short, which is ideal for highspeed applications. The planar design also permits IC-compatible gadgets. The carrier lifetime of this photodetector was in the sub-picosecond range, and it was frequently utilized for high-speed sampling. Due to the advancement of semiconductor material growth and manufacturing processes, a large amount of research and development work has been done in the area of MSM-PDs to find their uses and applications in many industries and operations. The addition of plasmonic-based nano-gratings to MSM-PDs, on the other hand, propelled MSM-PDs to new heights [23-46].

Recent studies have focused on the characteristics of metallic nano-gratings on the surface of MSM-PDs to enhance their light absorption capacities. For exceptional optical transmission (EOT) in nano-scale devices, stimulation of surface plasmon polaritons (SPPs) is required. The SPP is formed by the interaction of free charges (free electrons) on a metal surface with the electromagnetic field to which they are exposed. By altering the surface of MSM-PDs, it is feasible to increase the rate of light absorption. In contrast to traditional aperture theory (i.e., a typical type MSM-PDs), this trapped light has a greater order of magnitude and is responsible for the device's increased light absorption. SPPs play a crucial role in this progress since they contribute greatly to EOT. However, in-depth research is still happening on this issue. Metal nano-gratings (NGs), which are periodic structures, are used to accomplish this. The MSM-PDs can achieve massive light confinement, which is launched by surface plasmons due to the periodic structures (SPs). This is because the periodic structures on top of the device surface help in efficiently passing light into the devices, i.e., boost the device's light transmission efficiency. The optical characteristics of subwavelength NGs, which are homogeneous media determined by their form, or the geometry of the structures. Resonant reflection occurs in the device when the period of the nano-grating matches the wavelength of the incoming light. As a result, light can be trapped in very narrow spaces (or small areas). However, this trapped light has a higher order of magnitude in contrast to standard aperture theory (i.e., a conventional type MSM-PDs) and does the improvement of light absorption through stirring the SPPs into the devices [27-29,31-46].

Nano-structured features (gratings, and others) have also been reported to improve the efficiency of thin-film solar cells by increasing the light absorption coefficient. On top of the GaAs-based solar cell, several subwavelength nanostructures, such as rectangular, trapezoidal, and triangle forms, were created and the influence of each geometry was explored. Many research groups calculated and reported the best absorption performance for nanostructured gratings on thin-film solar cells, proving that having nano-structures on the top surface of the solar cell can result in less reflection and better absorption as the surface area for incident light interaction increases. Due to their wide range of applications in solar observations, UV astronomy, missile tracking, automation, short-range communication security, as well as environmental and biological research, metal-oxide-based see-through solar-blind photodetectors (also known as solar-blind deep ultraviolet (DUV-MSM-PDs) have piqued interest. Many scientists and research organizations across the world have used nanoparticles, nanoholes, and nanoslits to increase the light enhancement qualities of devices appropriate for a variety of sophisticated applications ranging from sensing to optical networks [36,42,47-60]. The current state and prospects of MSM-PDs were recently provided in a thorough review paper in which the authors addressed a large area towards the development of MSM-PDs, including varieties of materials, manufacturing processes, and performance characteristics [16]. S. Kashyap et al., on the other hand, have demonstrated plasmonic-based photodetectors (PDs) with a double layer of nano-gratings with optimal results at a certain wavelength useful for the night vision applications [46]. They have reported on the development of a plasmonic-supported photodetector with a double nanograting that enhanced light absorption with an optimal height of subwavelength 
aperture, where both the top and bottom layers of the grating contribute to light trapping. However, research into double-layer structures, manufacturing feasibility, and material availability is still restricted, necessitating additional research and analysis.

In this review article, we analyze different types of nano-grating structures that improve the light-capturing properties of MSM-PDs and the most recent advances in their structures (mainly the simulation aspects) that can be used to ameliorate the light absorption in MSM-PDs or advanced optoelectronic devices.

\section{Basic Structures of MSM-PDs and Nano-Grating Structures}

The fundamental construction of MSM-PD is shown in Figure 1 and it comprises two metallic electrodes that are placed onto a semiconductor material or substrate, forming Schottky connections. The primary concept of MSM-PD is to establish a Schottky barrier that drives material depletion at the surface.
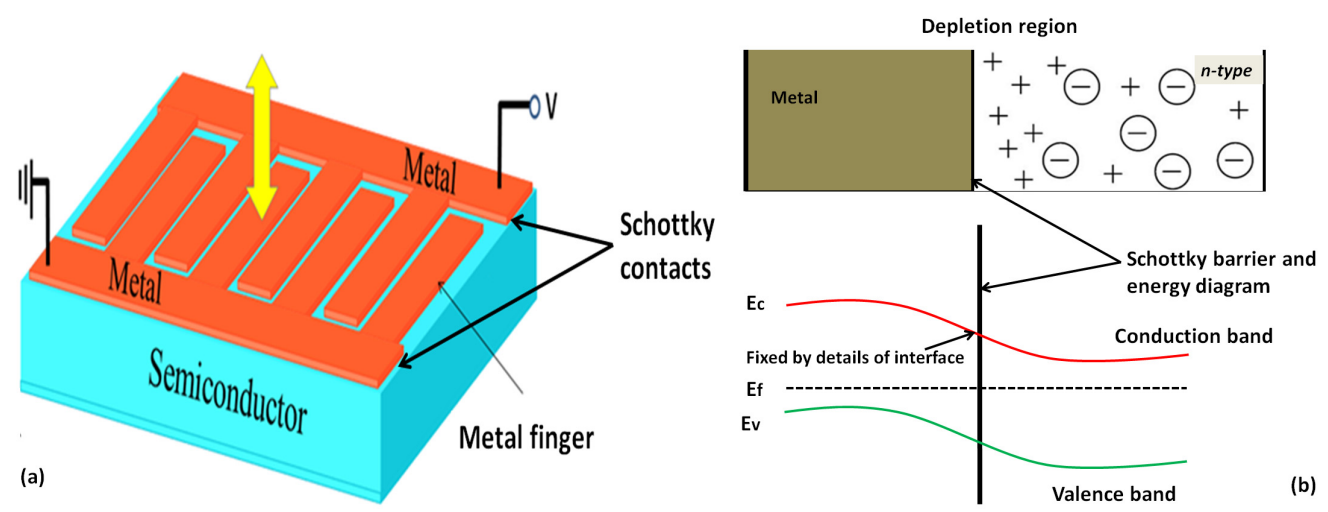

Figure 1. A typical MSM-PD structure contained two Schottky contacts deposited onto a semiconductor substrate and the cross-section of the metal area of the Schottky barrier and energy bandgap $[42,61]$.

These two Schottky electrodes are routinely interconnected in a comb configuration in an MSM-PD, leaving a free semiconductor surface between the two contacts that produce and form the active zone in which the light will be caught. The bias voltage was supplied between the two electrodes to disrupt the contacts' original electrical symmetry, resulting in one forward-biased Schottky junction and one reverse-biased Schottky junction, each yielding a wider space charge area. When light impinges on a semiconductor, it forms electric carriers (i.e., holes and electrons) that are collected by the electric field, resulting in a photocurrent. When compared to a Schottky photodiode or a PIN photodiode with the same quantum efficiency, its capacitance is reduced by around half. When compared to photodiodes, MSM-PD detection is quicker, and detection bandwidths may reach hundreds of gigahertz $(\mathrm{GHz})$, making it more appropriate for very high-speed optical communication devices [31-35,41-43].

Only the inorganic materials of III-V (GaAs, InGaAs) and IV ( $\mathrm{Si}, \mathrm{Ge})$ were the subject of early MSM-PD research. Researchers began to investigate MSM-PDs devices based on third-generation inorganic materials, such as III-V (GaN), IV $(\mathrm{SiC})$, metal oxides ( $\mathrm{ZnO}$, $\mathrm{TiO}_{2}, \mathrm{Ga}_{2} \mathrm{O}_{3}$ ), and other developing materials, as a result of the development of fiber optic communication technology and sensor systems (perovskites, 2D materials). Recently, a full evaluation of the research development for the aforesaid MSM-PDs (covering the fundamental structures and materials type, manufacturing processes, operating wavelengths, and performance characteristics) was presented in Refs. [16,18,62-71]. On the other hand, the choice of substrate materials is exclusively reliant on the kind of application and the wavelength-dependent responses that are required for the particular application. Figure 2 represents a schematic diagram that links all sections for the successful completion of the MSM-PDs study. 


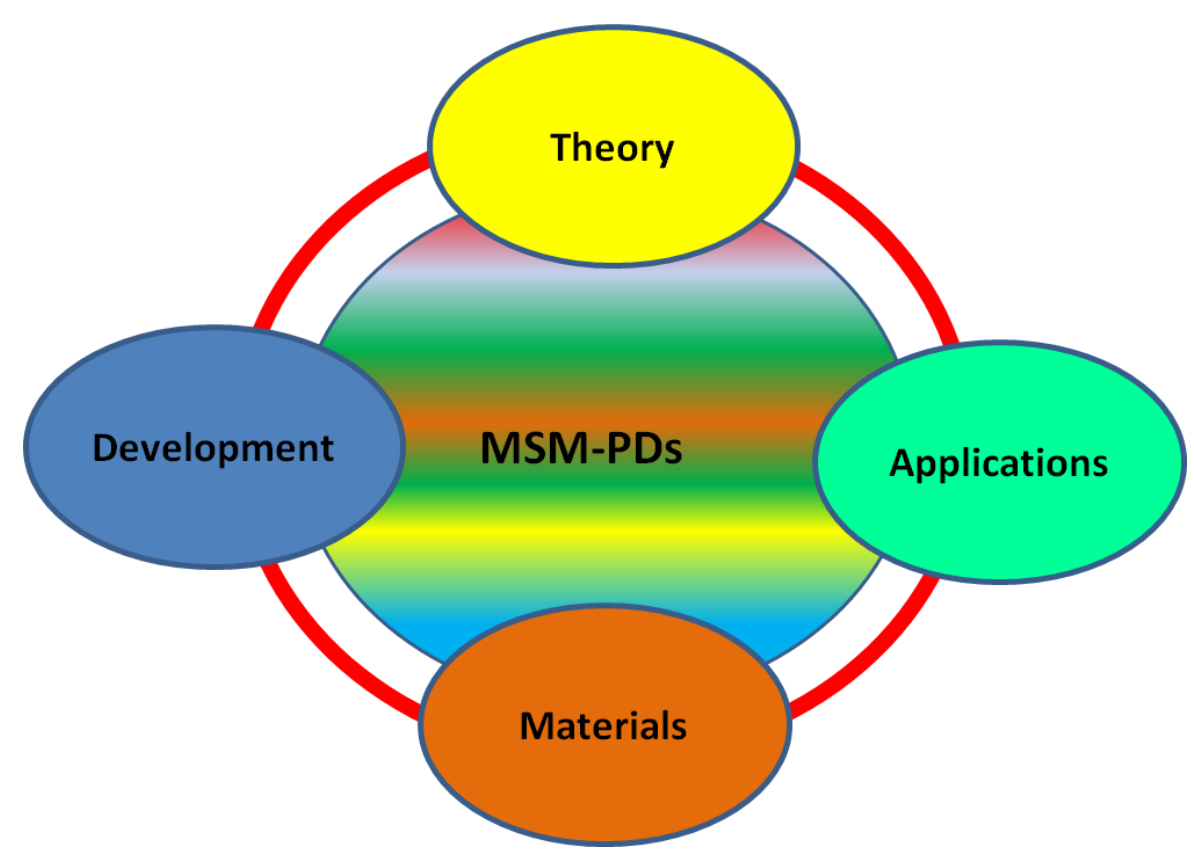

Figure 2. Schematic diagram that presents the relevant sections that are required to be focused on to complete the MSM-PDs study.

The advanced alternative for improving light absorption in MSM-PDs. The MSM-PDs with nano-gratings have enhanced optical qualities that are beneficial in speedier optical fiber communication systems, sensor networks, high-speed chip-to-chip interconnects, and high-speed sampling. Subwavelength nano-gratings can be thought of as a homogenous medium whose optical characteristics are governed by its geometry. The light wave may be resonant and reflect in the device structure when the nano-grating period is within the order of light wavelength, resulting in resonant reflection. Metals' photonic insulating qualities can be exploited to concentrate incident light in extremely tiny volumes or portions of a device. Light can be transmitted with a higher-order magnitude through subwavelength slits than through a standard aperture theory, which is a typical type of device. Figure 3 shows a typical MSM-PD structure with various grating structure forms and the produced grating structures on various substrates manufactured using two distinct techniques. The MSM-PD structures (Au grating/Au/GaAs) are made up of three layers (metal nanograting layer, unperturbed metal layer (under-layer) with conventional sub-wavelength apertures, and semiconductor (GaAs) substrate layer), as shown in Figure 3a. Many research groups have explored and optimized the performance (optical light absorption enhancement) of various types of nano-grating structures (including grating forms, height, breadth, and so on) for various upcoming photonics and optoelectronic applications. This article's discussion section has the most recent optimized findings. Fabrication and development of nano-structures of a certain design are always difficult in the disciplines of nanomaterials and thin films. However, advances in micro/nanofabrication processes, as well as major research efforts by academics and scientists, have opened additional possibilities for developing innovative and contemporary technologies. Figure $3 b$ shows images of photo-resist developed plasmonic metal nano-grating structures etched inside the top part of the Au layer using the focused ion beam (FIB) milling process, while Figure $3 c$ shows the images of practically developed photo-resist nano-grating structures together with the development process sequences. It is suggested that employing the FIB milling method to generate nano-gratings structure (i.e., particularly on top of metallic substrates) is preferable to traditional photolithography processes for cutting sharp-edge profiles [72]. 


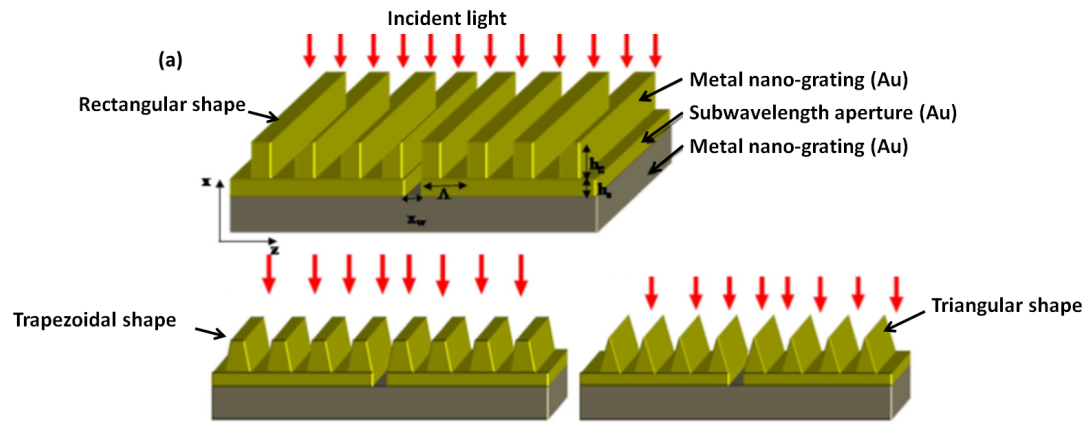

(b)

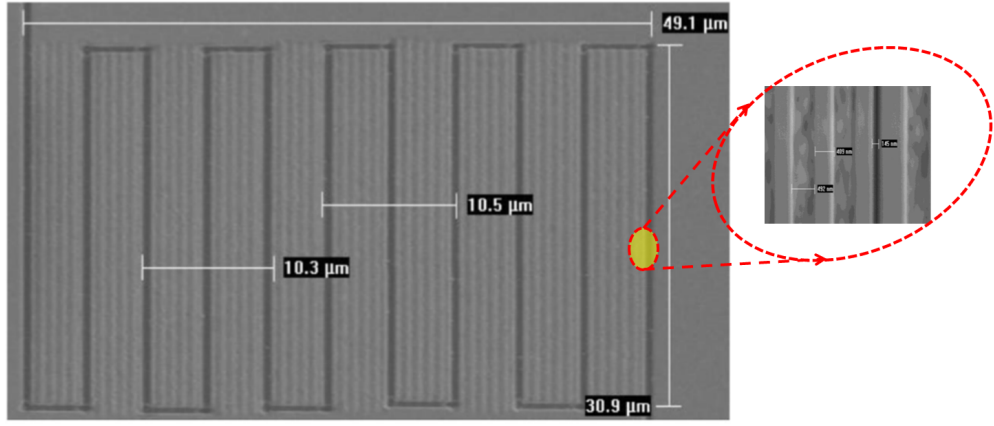

(c)

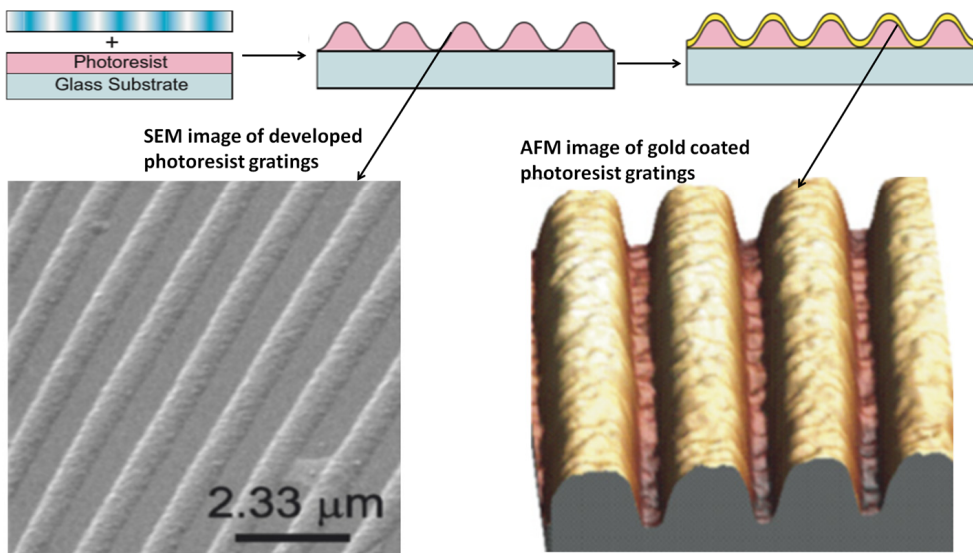

Figure 3. The schematic diagram for various types of nano-grating structures (a), SEM image of developed nano-grating structures prepared by using dual-beam FIB lithography process (b), and photo-resist nano-grating (uncoated and gold coated) structures developed on glass substrates by using simple spin-coating and sputtered deposition process, and their AFM and SEM images (c) $[44,72]$. Figure 3c, has been Adapted and reprinted from ref. 44, Copyright (2007), with permission from Elsevier B. V.

Light absorption is improved by catching and transferring more optical power to the device's absorbing active areas. The implementation or simulation of plasmonicsbased MSM-PDs using subwavelength slits and various shape gratings for various active materials and operating frequencies have been published in the literature, which will be highly useful for future research in this subject.

\section{Working Mechanism and Performance Parameters of MSM-PDs}

\subsection{Theoretical Aspects}

Light absorption is improved by catching and transferring more optical power to the device's absorbing active areas. The implementation or simulation of plasmonics-based MSM photodetectors using subwavelength slits and various shape gratings for various active materials and operating frequencies have been published in the literature, which are highly useful for future research in this theme. Aside from its adaptability and simplicity, another benefit of the FDTD approach is how simple it is to apply in code. Optiwave 
FDTD, also known as OptiFDTD, is a computer-aided design (CAD) and simulation tool that permits sophisticated passive and non-linear photonics components. Optiwave Inc. created the OptiFDTD software package to assist users in the design and simulation of complex passive and non-linear components using the FDTD approach [73]. Unlike other approaches, the FDTD method can represent wave propagation in complicated media such as dispersive, lossy, and non-linear media, as well as incorporate the effects of radiation and reflection [73,74]. The FDTD approach, like the BPM (beam propagation method), simulates the propagation of an incoming EM wave into a volume of space with a structure of interest, but instead of using a single-way wave equation like the BPM, it uses a set of Maxwell's equations. The FDTD technique is a direct solution to Maxwell's time-independent curl equations that address problems in both time and space dimensions before numerically resolving the resultant equations to generate magnetic and electric field distributions at every step. The primary program of the OptiFDTD software is based on the FDTD method, which solves both magnetic and electric fields in spatial and temporal domains with second-order numerical precision using the full-vector differential form of Maxwell's pair curl equations. The Lorentz-Drude model is used to develop the FDTD algorithm. As it is critical to identify the dielectric characteristics of materials to establish a good model, the Lorentz-Drude Model aids in determining the magnetic and dielectric properties of materials with nano-textured surfaces. While good conductor materials such as gold ( $\mathrm{Au})$ and silver $(\mathrm{Ag})$ have negative real parts of complex dielectric constants below plasma frequency, it is necessary to study thoroughly using multiple modes such as the LorentzDrude model and the frequency domain. Many scientists and researchers have studied and published in the literature the theoretical explanation of the working mechanism of MSM-PDs modeling using FDTD simulating tools (including Lorentz-Drude model, frequency domain, and other aspects) $[34,35,37,38,73-75]$. Figure 4 shows the working flow of MSM-PDs modeling using FDTD simulating tools together with the nano-structure grating assisted modeling feature, and various designs of nano-structure gratings.

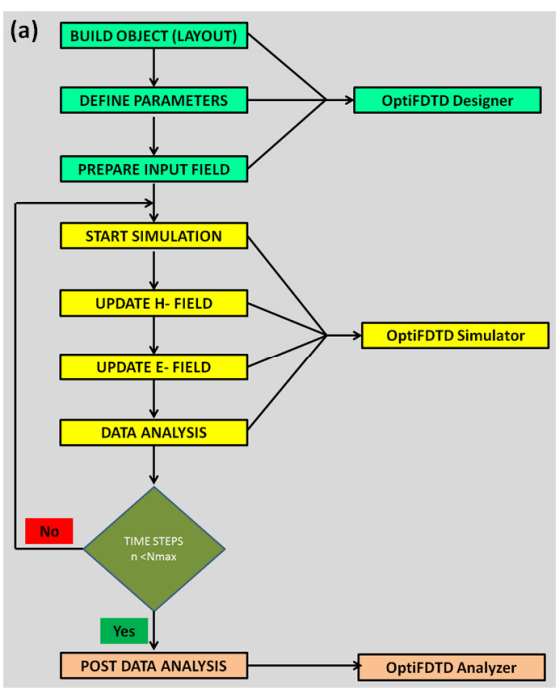

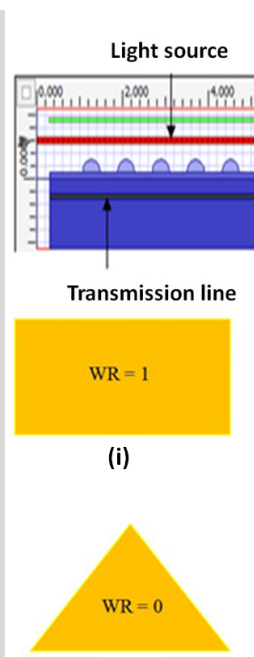

(iii)

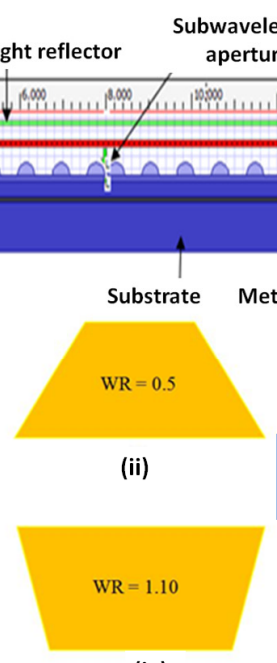

(iv) (b)

Figure 4. Schematic diagrams of the working flow of MSM-PDs modeling using FDTD simulating tools (a), nano-structure grating assisted modeling feature (b), and various designs of nano-structure grating with the definition of working ratio (WR) (i-Rectangular, ii-Trapezoidal, iii-Triangular, and iv invers Trapezoidal) (c) [76,77].

\subsection{Basic Working Mechanisms and Key Performance Parameters of MSM-PDs}

MSM-PDs are classified as Ohmic-contact type or Schottky contact type based on the type of heterojunction produced between the metal electrode and the semiconductor material, and their operating processes vary as well. Assume that two back-to-back Ohmic contacts are made between the semiconductor material and the metal electrodes for the 
Ohmic-contact type MSM-PDs. This mechanism differs based on the ambient mode, such as MSM-PD in the dark versus MSM-PD in the light exposure. Many publications, including a highly detailed review paper reported by L. Shi et al., explain the functioning mechanism of MSM-PDs under various settings [16]. When building an MSM-PD for any application, numerous essential performance aspects of photodetectors are always taken into account, including external quantum efficiency (EQE), responsivity, reaction time, light to dark current ratio, and detection capability.

\section{Advance States of Simulated Results and Discussion}

Due to the interesting applications in modern optoelectronic devices, the state-of-theart research activities on MSM-PDs are very wide. A significant number of research works have been conducted and reported by many research groups worldwide including the synthesis of suitable materials (i.e., organic, inorganic, doped, or undoped), development of multilayer thin-film structures of various dimensions such as nano-rods, nano-particles, and nano-gratings, etc., and characterization and investigation of their properties for various applications [1-25]. For example, the performance of MSM photodetectors on GaN was observed in the UV-spectral region and was reported to be increased by $50 \%$ with the insertion of $\mathrm{Al}$ nanoparticles in between the Schottky contacts of the photodetector. In addition, as compared to a standard MSM photodetector with merely a sub-wavelength aperture, an MSM detector with a sub-wavelength aperture enclosed by an aluminum (Al) nano-structured metal grating (while an Al-gratings based nano-antenna was integrated into a detector for photocurrent enhancement) demonstrated an eightfold increase in photocurrent enhancement (without grating). Figure 5 presents the design, development, and performance characteristics of an advanced MSM-PD that working in the UV region $[8,75]$.

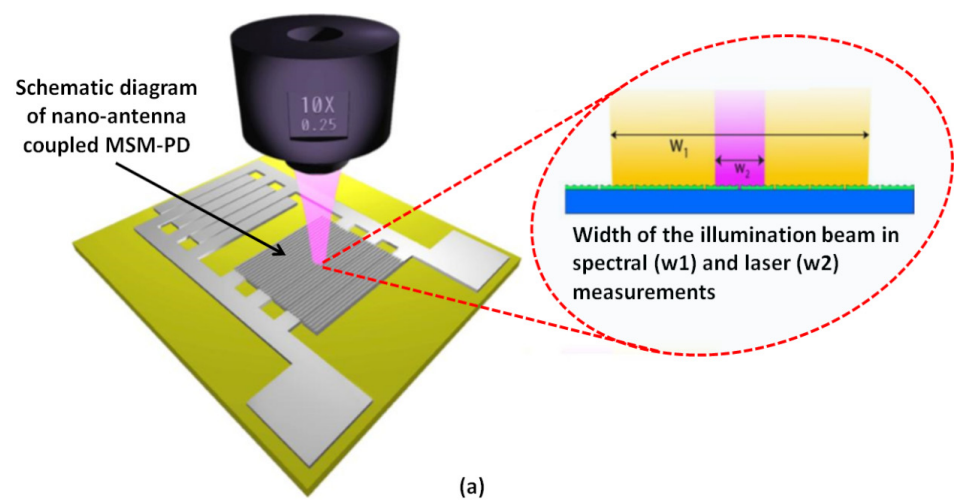

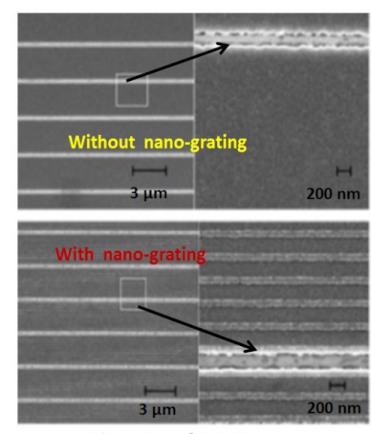

SEM images of MSM contacts (b)

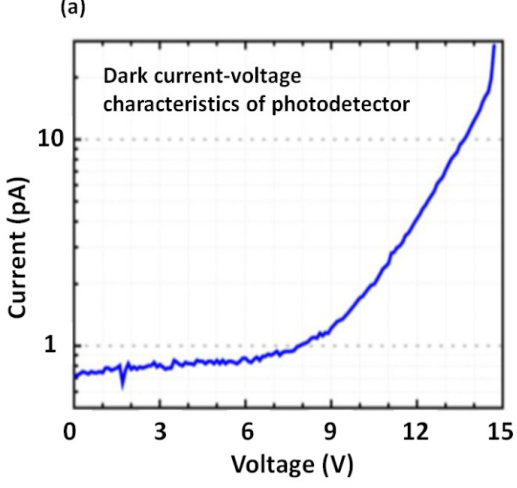

(c)

Figure 5. The concept, development, and characterization results of metal-gratings based nanoantenna that integrated into the detector for photocurrent enhancement. The schematic diagram of Al-nano-grating base nano-antenna coupled with MSM-PD (a), SEM images of developed contacts (b), and the measured dark-current performance (c) $[8,75]$. The figure has been reproduced with permission from Ref [75] (c) The Optical Society. 
Since 2011, Das et al., and his research group has been working on this very interesting and emerging topic of MSM-PD and have reported several modified structures in the literature to improve the MSM-PDs performances in terms of light capturing capabilities for modern optoelectronic devices. They have reported on the modeling of MSM-PD structures' light-capturing capability (in terms of light absorption enhancement) using various types (i.e., geometrical shapes) of metal nano-gratings. The light absorption improvement in MSM-PDs is heavily contingent on the nanograting forms, according to the simulation results. The rectangular nanograting structures can enable MSM-PDs to absorb incoming light (around $850 \mathrm{~nm}$ ) 50 times more efficiently than traditionally built MSM-PDs. However, this research group has continued to work on improving the light absorption of different aspects of MSM-PDs where a nano-structured of metal gratings has been placed onto the device's metal layer (simulation performances have been published numerous times) [33-35,37,38,41-43,76-78]. They have also studied the effects of nanograting profile (various width ratios, $0-1.10$ ), sub-wavelength aperture slit width, and grating thickness on light absorption enhancement by modeling and analyzing the features of MSM-PDs. They have written about the influence of geometrical structural differences in nano-grating structures, such as form, thickness, height, and breadth of slit-apertures, on the light absorption coefficient on the MSM-PD multiple times. Figure 6 shows an example of simulated findings, demonstrating that nano-gratings with a rectangular shape are more successful at increasing light absorption into the medium. Furthermore, the rectangular form of nanostructures is comparably easier to generate than any other shape in terms of the manufacturing procedure. The rectangular-shaped nano-grating profile had the highest light absorption enhancement factor (LAEF), which is defined by the ratio of normalized power transmittance with metal nano-grating to normalized power transmittance without nano-grating. The greatest LAEF performance was around 12.5, whereas triangular-shaped nano-gratings with $\mathrm{WR}=0$ had the lowest peak of LAEF, which was around 2.5 times. The simulation was run using constant values of $50 \mathrm{~nm}, 100 \mathrm{~nm}$, and $810 \mathrm{~nm}$ for the sub-wavelength aperture slit width, grating thickness, and period, respectively. The impact of nano-grating structures with different working ratios (WR ranging from 0 to 1 ) on the LAEF has also been explored [76].

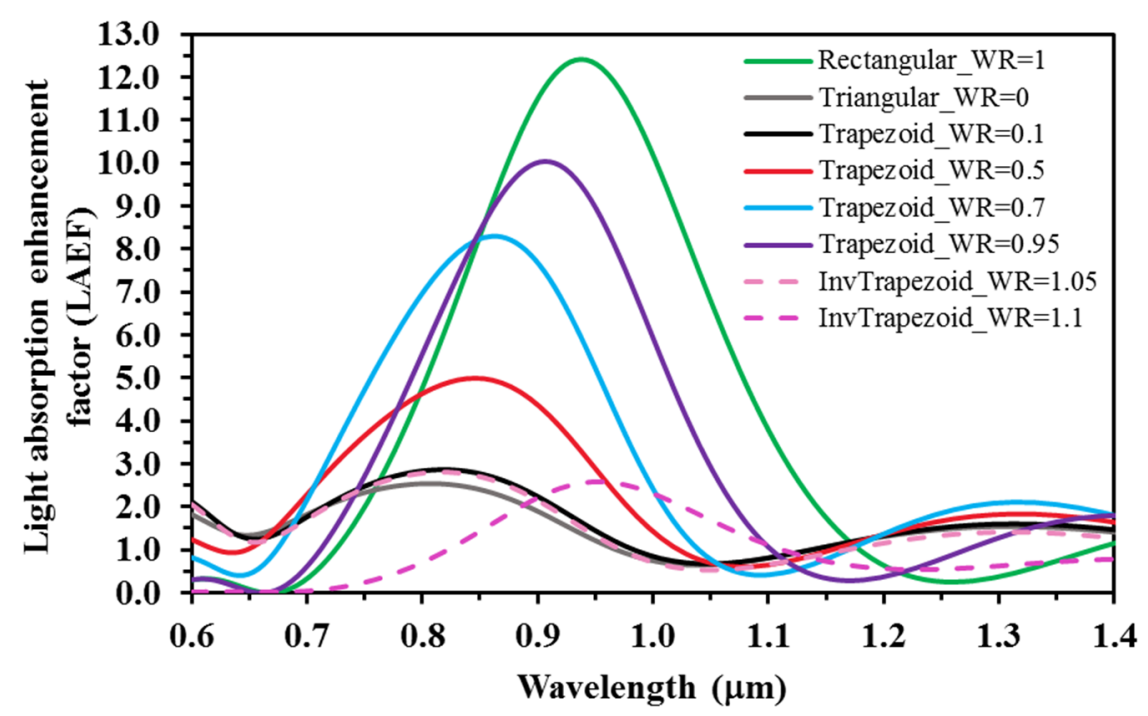

Figure 6. Light absorption enhancement factor (LAEF) spectrum of MSM-PD for various grating shapes. Note that $50 \%$ duty cycle, a constant of $100 \mathrm{~nm}$ grating thicknesses, and slit width of $50 \mathrm{~nm}$ were considered during the simulation process. The working ratios used for the nano-grating structures are presented in the figure legend [76].

The effect of sub-wavelength aperture slit width on LAEF was researched, and it was discovered that increasing the slit width resulted in a decrease in LAEF, as shown 
in Figure 7. The highest peak of LAEF for rectangular, triangular, and trapezoidal nanogratings can be reached at $50 \mathrm{~nm}$ slit width, but the greatest peak of LAEF for inverted trapezoidal nano-gratings profiles can be achieved at $150 \mathrm{~nm}$ slit width. The simulation research revealed that increasing the sub-wavelength aperture slit width resulted in a decrease in LAEF for all grating forms except the inverted trapezoidal. When the slit width was increased up to $100-150 \mathrm{~nm}$ for inverted trapezoidal geometries, the LAEF increased, but then progressively decreased.
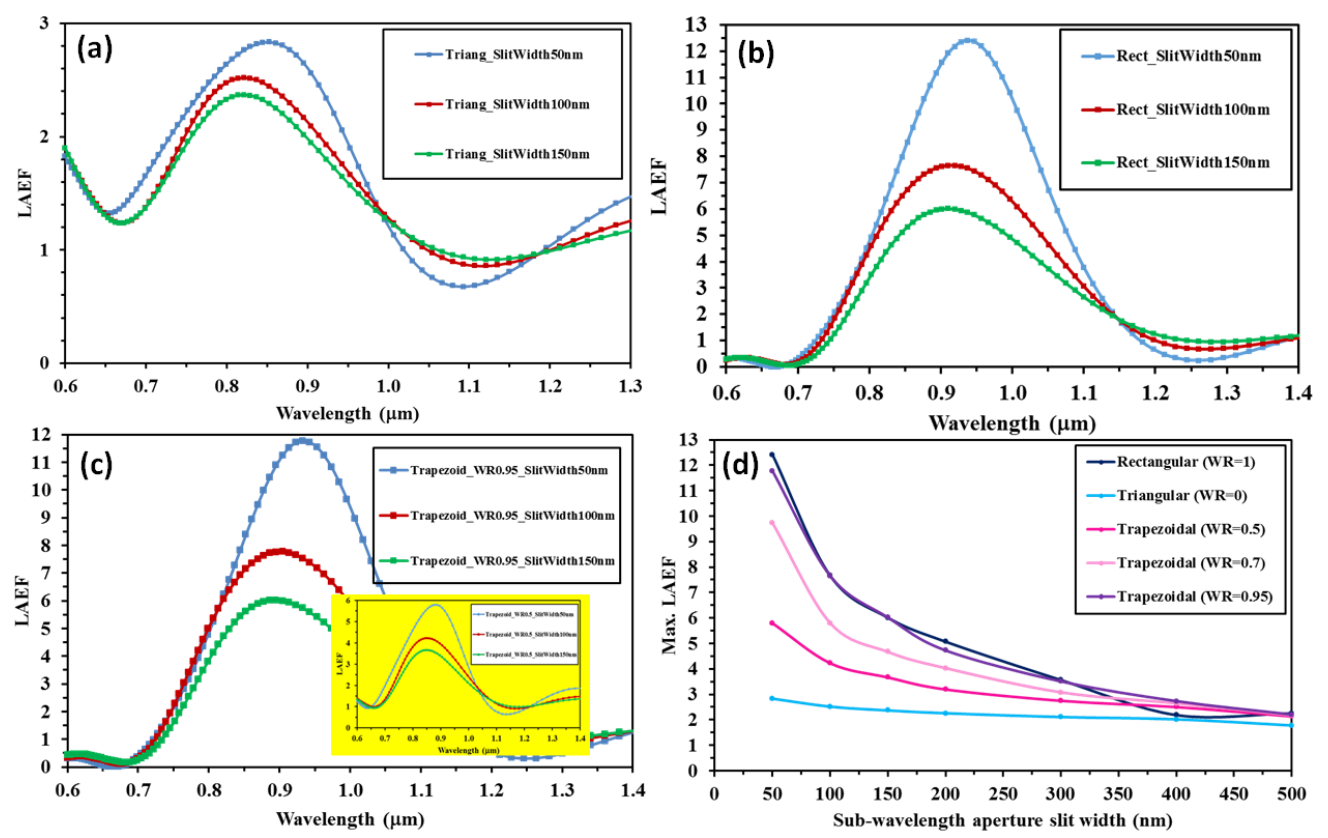

Figure 7. Light absorption enhancement factor (LAEF) spectrum of MSM-PD observed for various grating shapes with different slit-width, Traingel shape (a), Rectangular shape (b), Trapezoidal shape (c), and comparison of slit-width dependent maximum LAEF achieved for different nanograting structures (d) [76].

On the other hand, the LAEF was found to be completely dependent on the grating thickness (with higher grating thickness up to a certain nm thick layer) leading to increased light absorption enhancement. Figure 8 shows the maximum peak of LAEF reached by a rectangular-shaped nano-grating, which is $12.5 \%$ for $100 \mathrm{~nm}$ grating thickness, and the lowest peak achieved by a rectangular-shaped nano-grating, which was 2.5 for $30 \mathrm{~nm}$ grating thickness. If nanomaterials and nano/micro-manufacturing processes are accessible, an ideal device might be made with rectangular-shaped nano-gratings with $W R=1,50 \mathrm{~nm}$ sub-wavelength apertures slit width, and $100 \mathrm{~nm}$ grating thickness, according to the latest simulation results.

The majority of metal nanograting structures are made to be built on top of a thin gold layer. Due to its exceptional qualities, gold is the most preferred plasmonic material; yet gold is one of the most costly materials and might be difficult to source out sometimes. Silver has recently been reconsidered as a major material for the production of nanostructures because of its plasmonic capabilities. According to Ref. [46], the performance of plasmonic-based photodetectors (PDs) with a double layer of nanograting structures has been tuned for night vision applications at a wavelength of $1.4 \mathrm{~m}$. The quenching factor (QF) of 92.14 percent was found for the suggested design of plasmonic supported photodetector with double nanograting, confirming the augmentation in light with the optimal height of subwavelength aperture approximately $60 \mathrm{~nm}$ (Figure 9). 


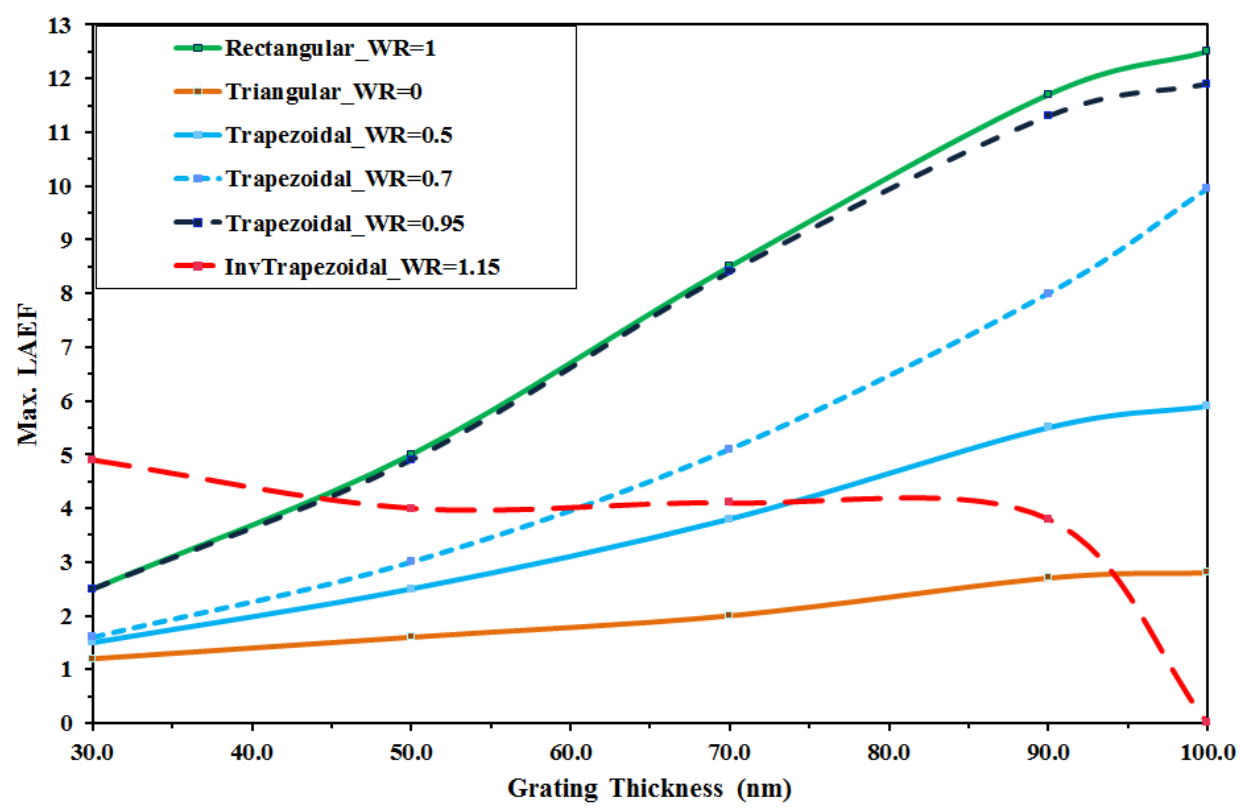

Figure 8. The possible maximum LAEF achieved in different nano-gratings thickness [76].

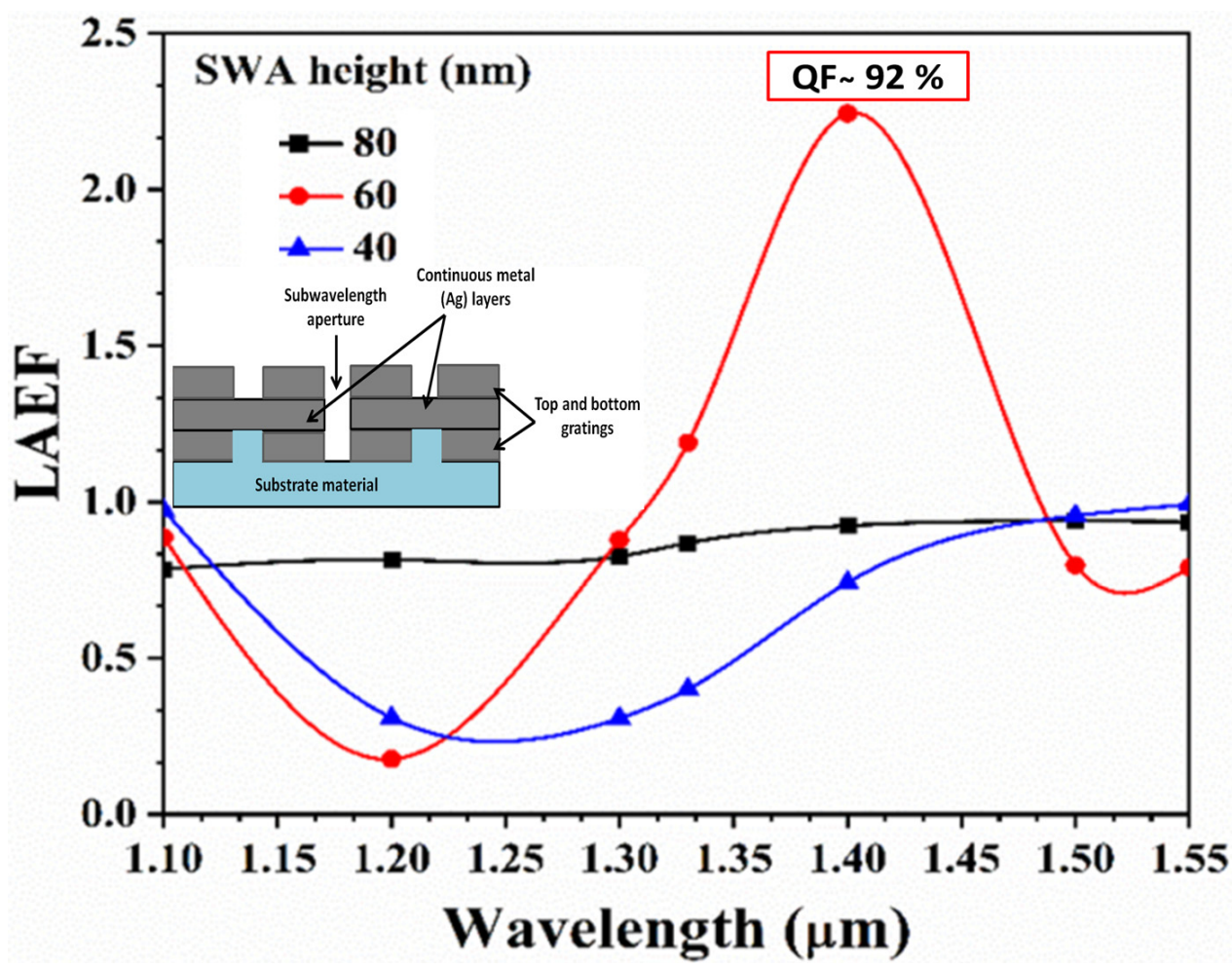

Figure 9. The schematic design and simulated performance characteristics of double silver grating MSM-PD [46].

However, putting this double layer nano-grating structure device into practice will be difficult owing to the disadvantages of employing silver as a top metal layer for any multilayer structure due to its rapid oxidation and surface deterioration. In such a situation, any other metal that may be employed as plasmonic materials would be preferable. NurE-Alam et al., recently published a paper on a silver-based composite material $(\mathrm{Ag}+\mathrm{SiC})$ that has reflective qualities similar to pure silver and can withstand high temperatures and ambient light exposure for a longer time [79]. This novel metallic composite material might be one of the greatest possibilities for future metal-based nano-structural device de- 
velopment, but it requires more research into nano-grating structure design and modeling for MSM-PDs as this contains a volumetric fraction of SiC. For the development of high current/temperature-driven modern optoelectronic devices, $\mathrm{SiC}$ and SiC-based materials are found to be highly recommended in the literature [79-83]. This new type of composite material can be useful for the design and development of modern devices including single/ multi-dimensional nano-structured high-temperature stable photodetectors for future applications.

\section{Conclusions}

We have presented the current state of nano-grating structures performances in supporting to improve the MSM-PDs that are precarious for the creation of future emergent optoelectronic devices for various applications. We have gone through the history and theoretical research that has been conducted so far for various types of single/multilayer metal-semiconductor/insulator-metal structures, as well as future work that can be implemented to improve the performance of nanostructured MSM-PDs. This article has given attention mainly to the latest update on the light absorption enhancement for MSM-PDs due to various nanostructured gratings and presented so far achieved for performance improvement. The practical development of nanostructures on MSM-PDs still requires more focused research including appropriate material suggestions and their fabrication processes.

Author Contributions: Conceptualization, M.N.-E.-A. and N.D.; software, A.I. and A.Z.M.A.; validation, M.N.-E.-A., A.I., A.Z.M.A., and N.D.; formal analysis, A.I., A.Z.M.A., and N.D.; investigation, M.N.-E.-A., A.I., A.Z.M.A., and N.D.; writing-original draft preparation, M.N.-E.-A.; writingreview and editing, M.N.-E.-A. and N.D.; visualization, M.N.-E.-A. and N.D.; supervision, N.D.; project administration, N.D.; funding acquisition, N.D. All authors have read and agreed to the published version of the manuscript.

Funding: This research is supported by the School of Engineering and Technology, Melbourne Campus, Victoria, Central Queensland University, Australia and the Centre for Intelligent Systems, Brisbane, QLD, Central Queensland University, Australia. This research is funded by the New Staff Research Grant RSH5171, 2019, School of Engineering and Technology, Central Queensland University, Australia.

Conflicts of Interest: The authors declare no conflict of interest.

\section{References}

1. Liu, M.Y.; Chou, S.Y. Nanoscale metal-semiconductor-metal photodetectors with sub-picosecond response time fabricated using electron beam lithography. J. Vac. Sci. Technol. B 1992, 10, 2932-2935. [CrossRef]

2. Huang, Z. Multi Gigahertz Indium Gallium Arsenide/Indium Phosphide Inverted MSM Photodetectors for Photoreceiver and Waveguide Applications. Ph.D. Thesis, Georgia Institute of Technology, Atlanta, GA, USA, 15 November 2003.

3. Das, N. Nanostructured Photodetector for Enhanced Light Absorption. Computational Nanotechnology Using Finite Difference Time Domain; CRC Press: Boca Raton, FL, USA, 2013; pp. 251-290.

4. Dahal, R. Fabrication and Characterization of III-Nitride Nanophotonic Devices. Ph.D. Thesis, Kansas State University, Manhattan, KS, USA, 2009.

5. Smith, F.W.; Le, H.Q.; Diadiuk, V.; Hollis, M.A.; Calawa, A.R.; Gupta, S.; Frankel, M.; Dykaar, D.R.; Mourou, G.A.; Hsiang, T.Y. Picosecond GaAs-based photoconductive optoelectronic detectors. Appl. Phys. Lett. 1989, 54, 890-892. [CrossRef]

6. Darweesh, A.A. Optical Enhancement in Periodic Plasmonic Gratings for SERS and Metal-Semiconductor-Metal Photodetectors (MSM-PDs) Applications. Ph.D. Thesis, University of Arkansas, Fayetteville, AR, USA, 2019; p. 3182.

7. Hetterich, J.; Bastin, G.; Gippius, N.A.; Tikhodeev, S.G.; von Plessen, G.; Lemmer, U. Optimized design of plasmonic MSM photodetector. IEEE J. Quantum. Electron. 2007, 43, 855-859. [CrossRef]

8. Cinel, N.A. EBL Fabricated Plasmonic Nanostructures for Sensing Applications. Ph.D. Thesis, Bilkent University, Ankara, Turkey, 2013.

9. Shen, L. Ultrafast Photodetector on the InP-Membrane-on-Silicon Platform. Ph.D. Thesis, Technische Universiteit Eindhoven, Eindhoven, The Netherlands, 10 November 2016.

10. BenMoussa, A.; Hochedez, J.F.; Dahal, R.; Li, J.; Lin, J.Y.; Jiang, H.X.; Soltani, A.; DeJaeger, J.-C.; Kroth, U.; Richter, M. Characterization of AIN metal-semiconductor-metal diodes in the spectral range of 44-360 nm: Photoemmission assessments. Appl. Phys. Lett. 2008, 92, 022108. [CrossRef] 
11. Hasan, R.M. Metal Oxide, Group V-VI Chalcogenides and GAN/ALGAN Photodetectors. Ph.D. Thesis, George Mason University, Fairfax, VA, USA, 2016.

12. Sze, S.M.; Li, Y.; Ng, K.K. Physics of Semiconductor Devices, 3rd ed.John Wiley \& Sons, Inc.: Hobboken, NJ, USA, 2007.

13. Nasir, A.; Jung-Wook, M.; Chun Hong, K.; Abdullah, A.; Davide, P.; Ram Chandra, S.; Malleswararao, T.; Tien Khee, N.; Boon, S.O. Deep-ultraviolet integrated photonic and optoelectronic devices: A prospect of the hybridization of group III- nitrides, III-oxides, and two-dimensional materials. J. Semiconductors 2019, 40, 121801. [CrossRef]

14. Dahal, R.; Li, J.; Fan, Z.Y.; Nakarmi, M.L.; Al Tahtamouni, T.M.; Lin, J.Y.; Jiang, H.X. AlN MSM and Schottky photodetectors. Physica Status Solidi C 2008, 5, 2148-2151. [CrossRef]

15. Cai, Y.; Shen, S.; Zhu, C.; Zhao, X.; Bai, J.; Wang, T. Nonpolar (1120) GaN metal-semiconductor-metal photodetectors with superior performance on silicon. ACS Appl. Mater. Interfaces 2020, 12, 25031-25036. [CrossRef] [PubMed]

16. Shi, L.; Chen, K.; Zhai, A.; Li, G.; Fan, M.; Hao, Y.; Zhu, F.; Zhang, H.; Cui, Y. Status and Outlook of Metal-Inorganic Semiconductor-Metal Photodetectors. Laser Photonics Rev. 2020, 15, 2000401. [CrossRef]

17. Casalino, M.; Iodice, M.; Sirleto, L.; Rao, S.; Rendina, I.; Coppola, G. Low dark current silicon-on-insulator waveguide metalsemiconductor-metal-photodetector based on internal photoemissions at $1550 \mathrm{~nm}$. J. Appl. Phys. 2013, 114, 153103. [CrossRef]

18. Sugeta, T.; Urisu, T. WP-B2 high-gain metal—Semiconductor-Metal photodetectors for high-speed optoelectronic circuits. IEEE Trans. Electron Devices 1979, 26, 1855-1856. [CrossRef]

19. Figueroa, L.; Slayman, C. A novel heterostructure interdigital photodetector (HIP) with picosecond optical response. IEEE Electron Device Lett. 1981, 2, 208-210. [CrossRef]

20. Wei, C.; Klein, H.-J.; Beneking, H. Symmetrical Mott barrier as a fast photodetector. Electron. Lett. 1981, 17, 688-690. [CrossRef]

21. Slayman, C.; Figueroa, L. Frequency and pulse response of a novel high-speed interdigital surface photoconductor (IDPC). IEEE Electron Device Lett. 1981, 2, 112-114. [CrossRef]

22. Ghosh, S.; Lin, K.-C.; Tsai, C.-H.; Kumar, H.; Chen, Q.; Zhang, L.; Son, B.; Tan, C.S.; Kim, M.; Mukhopadhyay, B.; et al. Metal-Semiconductor-Metal GeSn Photodetectors on Silicon for Short-Wave Infrared Applications. Micromachines 2020, 11, 795. [CrossRef] [PubMed]

23. Kashyap, R.; Nemova, G. Surface Plasmon Resonance-Based Fiber and Planar Waveguide Sensors. J. Sens. 2009, $2009,1-9$. [CrossRef]

24. Sano, E. A device model for metal-semiconductor-metal photodetectors and its applications to optoelectronic integrated circuit simulation. IEEE Trans. Electron Devices 1990, 37, 1964-1968. [CrossRef]

25. Burm, J.; Litvin, K.I.; Schaff, W.J.; Eastman, L.F. Optimization of high-speed metalsemiconductor-metal photodetectors. IEEE Photonics Technol. Lett. 1994, 6, 722-724. [CrossRef]

26. Nusir, A.I.; Hill, A.M.; Manasreh, M.O.; Herzog, J.B. Near-infrared metalsemiconductor-metal photodetector based on semiinsulating GaAs and interdigital electrodes. Photonics Res. 2015, 3, 1-4. [CrossRef]

27. Das, N.; Karar, A.; Vasiliev, M.; Tan, C.L.; Alameh, K.; Lee, Y.T. Analysis of nano-grating-assisted light absorption enhancement in metal-semiconductor-metal photodetectors patterned using focused ion-beam lithography. Opt. Commns. 2011, 284, 1694-1700. [CrossRef]

28. Karar, A.; Das, N.; Tan, C.L.; Alameh, K.; Lee, Y.T.; Karouta, F. High-responsivity plasmonics-based GaAs metal-semiconductormetal photo-detectors. Appl. Phys. Lett. 2011, 99, 133112. [CrossRef]

29. Tan, C.L.; Lysak, V.V.; Das, N.; Karar, A.; Alameh, K.; Lee, Y.T. Absorption enhancement of MSM photo-detector structure with a plasmonic double grating structure. In Proceeding of the 2010 10th IEEE Conference on Nanotechnology (IEEE-NANO 2010), Seoul, Korea, 17-20 August 2010.

30. Soole, J.B.D.; Schumacher, H. InGaAs metal-semiconductor-metal photodetectors for long wavelength optical communication. IEEE J. Quantum Electron. 1991, 27, 737-752. [CrossRef]

31. Guo, J.; Tu, Y.; Yang, L.; Wang, L.; Wang, B. Design of a double grating-coupled surface plasmon color filter. In Proceedings of the Optical Components and Materials XIII, San Francisco, CA, USA, 15-17 February 2016; SPIE: Bellingham, WA, USA, 2016; Volume 9744, p. 97440C.

32. Xiong, X.H.; Zhan, L.M.; Ke, X. Effects of grating slant angle on surface plasmon resonance and its applications for sensors. Appl. Mech. Mater. 2014, 536, 342-345. [CrossRef]

33. Masouleh, F.F.; Das, N.; Mashayekhi, H.R. Comparison of different plasmonic nano-grating profiles for quality light absorption in nanostructured metal-semiconductor-metal photodetectors. Opt. Eng. 2013, 12, 45-46.

34. Das, N.; Masouleh, F.F.; Mashayekhi, H.M. Light absorption and reflection in nanostructured GaAs metal-semiconductor-metal photo-detectors. IEEE Trans. Nanotech. 2014, 13, 982-989. [CrossRef]

35. Masouleh, F.F.; Das, N.; Mashayekhi, H.R. Assessment of amplifying effects of ridges spacing and height on nano-structured MSM photo-detectors. Opt. Quantum Electron. 2015, 47, 193-201. [CrossRef]

36. Le, K.Q.; Ngo, Q.M.; Nguyen, T.K. Nanostructured metal-insulator-metal metamaterials for refractive index biosensing applications: Design, fabrication, and characterization. IEEE J. Sel. Top Quantum Electron. 2016, 23, 388-393. [CrossRef]

37. Masouleh, F.F.; Das, N.; Mashayekhi, H.R. Optimization of light transmission efficiency for nano-grating assisted MSM-PDs by varying physical parameters. Phot. Nano Fund. Appl. 2014, 12, 45-53. [CrossRef]

38. Das, N.; Karar, A.; Tan, C.L.; Alameh, K.; Lee, Y.T. Impact of Nanograting Phase-Shift on Light Absorption Enhancement in Plasmonics-Based Metal-Semiconductor-Metal Photodetectors. Adv. Opt. Technol. 2011, 2011, 1-8. [CrossRef] 
39. Fan, Z.; Su, Y.; Zhang, H.; Han, X.; Ren, F. Analysis of aluminum nano-gratings assisted light reflection reduction in GaAs metal-semiconductor-metal photodetectors. In Proceedings of the SPIE Optics + Photonics for Sustainable Energy, San Diego, CA, USA, 9-13 August 2015; The International Society for Optics and Photonics (SPIE): Bellingham, WA, USA, 2015; p. 95630T.

40. Darweesh, A.A.; Bauman, S.J.; Herzog, J.B. Improved optical enhancement using double-width plasmonic gratings with nanogaps. Photonics Res. 2016, 4, 173-180. [CrossRef]

41. Masouleh, F.F.; Das, N.; Rozati, S.M. Optimal subwavelength design for efficient light trapping in central slit of plasmonics-based metal-semiconductor-metal photo-detectors. Opt. Quant. Electron. 2015, 47, 1477-1485. [CrossRef]

42. Chandan, N.; Das, N.; Masouleh, F.F. Analysis of plasmonics-based nano-structured MSM-PDs for enhanced light absorption. In Proceeding of the 2017 Eleventh International Conference on Sensing Technology (ICST), Sydney, NSW, Australia, 4-6 December 2017; pp. 1-5.

43. Masouleh, F.F.; Rozati, S.M.; Das, N. Performance improvement of plasmonic-based thin film assisted MSM-PDs. Optik 2018, 157, 733-742. [CrossRef]

44. Massenot, S.; Chevallier, R.; Parriaux, O. Tunable grating-assisted surface plasmon resonance by use of nano-polymer dispersed liquid crystal electro-optical material. Optics Commun. 2007, 275, 318-323. [CrossRef]

45. García-Martín, A.; Ward, D.R.; Natelson, D.; Cuevas, J.C. Field enhancement in subnanometer metallic gaps. Phys. Rev. B 2011, 83, 193404. [CrossRef]

46. Kashyap, S.; Kaur, H. Enhancement of Light Absorption in Plasmonic Based Photodetector with Double Nanograting Structure. Available online: https: / / assets.researchsquare.com/files/rs-505093/v1/0d1d274f-78b6-406c-b7fa-30be5ebf4a6f.pdf?c=1631 882990 (accessed on 27 November 2021).

47. Ding, M.; Guo, Z.; Chen, X.; Ma, X.; Zhou, L. Surface/Interface Engineering for Constructing Advanced Nanostructured Photodetectors with Improved Performance: A Brief Review. Nanomaterials 2020, 10, 362. [CrossRef]

48. Savoia, S.; Ricciardi, A.; Crescitelli, A.; Granata, C.; Esposito, E.; Galdi, V.; Cusano, A. Surface sensitivity of Rayleigh anomalies in metallic nanogratings. Opt. Express 2013, 21, 23531-23542. [CrossRef] [PubMed]

49. Darweesh, A.A.; Bauman, S.J.; Brawley, Z.T.; Herzog, J.B. Improved optical enhancement in binary plasmonic gratings with nanogap spacing. In Proceedings of the Nanoengineering: Fabrication, Properties, Optics, and Devices XIII, San Diego, CA, USA, 30-31 August 2016; p. 99270Z.

50. Abdulhalim, I.; Zourob, M.; Lakhtakia, A. Surface plasmon resonance for biosensing: A mini-review. Electromagnetics 2008, 28, 214-242. [CrossRef]

51. Khan, F.; Khan, W.; Kim, S.-D. High-Performance Ultraviolet Light Detection Using Nano-Scale-Fin Isolation AlGaN/GaN Heterostructures with ZnO Nanorods. Nanomaterials 2019, 9, 440. [CrossRef] [PubMed]

52. Khan, W.; Ajmal, H.; Khan, F.; Huda, N.; Kim, S.-D. Induced photonic response of ZnO Nanorods Grown on oxygen plasmatreated seed crystallites. Nanomaterials 2018, 8, 371. [CrossRef] [PubMed]

53. Alenezi, M.R.; Henley, S.J.; Silva, S.R.P. On-chip fabrication of high performance nanostructured ZnO UV detectors. Sci. Rep. 2015, 5, 8516. [CrossRef] [PubMed]

54. Oener, S.Z.; van de Groep, J.; Macco, B.; Bronsveld, P.C.P.; Kessels, W.M.M.; Polman, A.; Garnett, E.C. Metal-InsulatorSemiconductor Nanowire Network Solar Cells. Nano Lett. 2016, 16, 3689-3695. [CrossRef]

55. Fadakar Masouleh, F.; Das, N.; Rozati, S.M. Nano-Structured Gratings for Improved Light Absorption Efficiency in Solar Cells. Energies 2016, 9, 756. [CrossRef]

56. Shalev, E.; Oksenberg, E.; Rechav, K.; Popovitz-Biro, R.; Joselevich, E. Guided CdSe Nanowires Parallelly Integrated into Fast Visible-Range Photodetectors. ACS Nano 2017, 11, 213-220. [CrossRef] [PubMed]

57. Lee, M.; Son, H.; Lee, H.-Y.; e Moon, J.; Kim, H.; Park, J.-I.; Liu, Z.; Hahm, M.G.; Yang, M.; Kim, U.J. Nanovoid-driven high crystalline aluminum nitride and its application in solar blind UV photodetectors. J. Mater. Chem. C 2020, 8, 14431-14438. [CrossRef]

58. Wei, T.-C.; Tsai, D.-S.; Ravadgar, P.; Ke, J.-J.; Tsai, M.-L.; Lien, D.-H.; Huang, C.-Y.; Horng, R.-H.; He, J.-H. See-through Ga $2 \mathrm{O}_{3}$ solar-blind photodetectors for use in harsh environments. IEEE J. Sel. Topics Quantum Electron. 2014, $20,3802006$.

59. Iqbal, T.; Tehseen, A.; Bashir, A.; Afsheen, S.; Tahir, M.B.; Abrar, M. Study of plasmonic bandgap of optimization of geometrical parameters of metallic grating devices. Solid State Commun. 2021, 327, 114212. [CrossRef]

60. Ding, Y.; Yoon, J.; Javed, M.H.; Song, S.H.; Magnusson, R. Mapping Surface-Plasmon Polaritons and Cavity Modes in Extraordinary Optical Transmission. IEEE Photonics J. 2011, 3, 365-374. [CrossRef]

61. Al-Azzawi, A. Photonics: Principles and Practices; CRC Press: Boca Raton, FL, USA, 2006.

62. Wang, S.Y.; Bloom, D.M. $100 \mathrm{GHz}$ bandwidth planar GaAs Schottky photodiode. Electron. Lett. 1983, 19, 554. [CrossRef]

63. Roth, W.; Schumaher, H.; Kluge, J.; Geelen, H.J.; Beneking, H. The DSI diode-A fast large-area optoelectronic detector. IEEE Trans. Electron Devices 1985, 32, 1034. [CrossRef]

64. Chen, X.; Wang, D.; Wang, T.; Yang, Z.; Zou, X.; Wang, P.; Luo, W.; Li, Q.; Liao, L.; Hu, W.; et al. Enhanced photoresponsivity of a GaAs nanowire metal-semiconductor-metal photodetector by adjusting the fermi level. ACS Appl. Mater. Interfaces 2019, 11, 33188. [CrossRef] [PubMed]

65. Kalinina, E.V.; Lebedev, A.A.; Kozlovski, V.V.; Zabrodskiy, V.V.; Strelchuk, A.M.; Nikitina, I.P. Electrophysical and Optical Properties of 4H-SiC UV Detectors Irradiated with Electrons. Mater. Sci. Forum 2019, 963, 722. [CrossRef] 
66. Walker, D.; Monroy, E.; Kung, P.; Wu, J.; Hamilton, M.; Sanchez, F.J.; Diaz, J.; Razeghi, M. High-speed, low-noise metalsemiconductor-metal ultraviolet photodetectors based on GaN. Appl. Phys. Lett. 1999, 74, 762. [CrossRef]

67. Afzal, N.; Devarajan, M.; Ibrahim, K. Growth of AlInN film on GaAs substrate and its application to MSM UV photodetector. Mater. Res. Express 2016, 3, 085904. [CrossRef]

68. Ahmed, A.A.; Hashim, M.R.; Abdalrheem, R.; Rashid, M. High-performance multicolor metal-semiconductor-metal Si photodetector enhanced by nanostructured $\mathrm{NiO}$ thin film. J. Alloys Compd. 2019, 798, 300. [CrossRef]

69. Shi, L.; Cui, Y.; Gao, Y.; Wang, W.; Zhang, Y.; Zhu, F.; Hao, Y. High performance ultrathin MoO3/Ag transparent electrode and its application in semitransparent organic solar cells. Nanomaterials 2018, 8, 473.

70. Chen, M.W.; Chen, C.Y.; Lien, D.H.; Ding, Y.; He, J.H. Photoconductive enhancement of single ZnO nanowire through localized Schottky effects. Opt. Express 2010, 18, 14836.

71. Li, X.; Chen, X.; Xu, X.; Hu, X.; Zuo, Z. Enhanced Performance of a Visible Light Detector Made with Quasi-Free-Standing Graphene on SiC. Materials 2019, 12, 3227.

72. Das, N.; Karar, A.; Tan, C.L.; Vasiliev, M.; Alameh, K.; Lee, Y.T. Metal-semiconductor-metal (MSM) photodetectors with plasmonic nanogratings. Pure Appl. Chem. 2011, 83, 2107-2113. [CrossRef]

73. Opti-FDTD. OptiFDTD Technical Background and Tutorials, 12th ed.; Optiwave: Ottawa, ON, Canada, 2014.

74. Hasan, I.M. FDTD Analysis of Plasmonic and Nanojet Enhanced Photodetectors for Improved Performance. Master's Thesis, The University of New Mexico, Albuquerque, NM, USA, 2012.

75. Bütün, S.; Cinel, N.A.; Özbay, E. Nano-antenna coupled UV subwavelength photodetectors based on GaN. Opt. Exp. 2012, 20, 2649-2656. [CrossRef]

76. Maslihan, A.Z. Modelling and Analysis of Nano-Structured Metal-Semiconductor-Metal Photo-Detectors for Enhanced Light Absorption. Bachelor's Thesis, Curtin University, Perth, WA, Australia, 2014.

77. Islam, A.; Uzzal, M.; Das, N.; Hasan, M. Modeling and Analysis of Different Nano-gratings to Enhance Light Absorption in MSM-PDs. In Proceedings of the 2019 International Conference on Robotics, Electrical and Signal Processing Techniques (ICREST), Dhaka, Bangladesh, 10-12 January 2019; pp. 369-373.

78. Islam, A.; Das, N.; Uzzal, M.M. A Comprehensive Study on the Impact of Various Nano-gratings on MSM-PDs for Enhancement in the Light Absorption. AIUB J. Sci. Eng. 2019, 18, 27-34. [CrossRef]

79. Basher, M.K.; Vasiliev, M.; Das, N. Physical Vapor-Deposited Silver (Ag)-Based Metal-Dielectric Nanocomposites for Thin-Film and Coating Applications. Appl. Sci. 2021, 11, 6746. [CrossRef]

80. Chang, W.R.; Fang, Y.K.; Ting, S.F.; Tsair, T.S.; Chang, C.N.; Lin, C.Y.; Chen, S.F. The hetero-epitaxial SiCN/Si MSM photodetector for high-temperature deep-UV detecting applications. IEEE Electron. Device Lett. 2003, 24, 565-567. [CrossRef]

81. Juang, F.R.; Fang, Y.K.; Chiang, Y.T.; Chou, T.H.; Cheng, I.L. A high-performance n-i-p SiCN homojunction for low-cost and high-temperature ultraviolet detecting applications. IEEE Sens. J. 2011, 11, 150-154. [CrossRef]

82. Sang, L.; Liao, M.; Sumiya, M. A Comprehensive Review of Semiconductor Ultraviolet Photodetectors: From Thin Film to One-Dimensional Nanostructures. Sensors 2013, 13, 10482-10518. [CrossRef] [PubMed]

83. Amorim, M.; Savio, R.; Massi, M.; Santiago, H. Applications of SiC-Based Thin Films in Electronic and MEMS Devices. In Physics and Technology of Silicon Carbide Devices; InTech: London, UK, 2012. 